NEVKP could
result in better
quality grafts
and might
offer the
opportunity
to assess
grafts prior to
transplantation

TRANSPLANTATION

\title{
Continuous normothermic ex vivo perfusion improves kidney graft function
}

Pressure-controlled continuous normothermic ex vivo kidney perfusion (NEVKP) improves renal graft function in pigs, say researchers. Markus Selzner and colleagues suggest that use of this preservation technique might reduce rates of delayed graft function and improve outcomes in kidney transplantation. "We aim to maintain physiologic conditions during NEVKP," says Selzner. "The kidneys are kept at $37^{\circ} \mathrm{C}$ and supplied with nutrition and oxygen using a perfusion solution with erythrocytes and a normal oncotic pressure. The conditions are chosen so that the kidney functions ex vivo as it would in the body."

Using pig autotransplantation models of heart-beating donation (HBD) and donation after circulatory death (DCD), the researchers showed that $8 \mathrm{~h}$ of NEVKP maintained graft function without preservation injury. In the HBD model, serum creatinine and blood urea nitrogen (BUN) levels at day 10 posttransplantation were similar to baseline in NEVKP graft recipients, whereas recipients of grafts that had undergone static cold storage
(SCD) had increased serum creatinine and BUN levels. In the DCD model, NEVPK graft recipients had significantly better post-transplantation kidney function than SCD graft recipients, as indicated by serum creatinine, BUN and potassium levels. In both models, animal survival was similar in SCD and NEVPK groups and histological analysis showed no differences in graft tubular injury, interstitial inflammation, oedema or fibrosis.

"NEVKP could result in better quality grafts and might offer the opportunity to assess grafts prior to transplantation," concludes Selzner.

Ellen F. Carney

ORIGINAL ARTICLES Moritz, K. J. et al. Continuous normothermic ex vivo kidney perfusion improves graft function in donation after circulatory death pig kidney transplantation. Transplantation http://dx.doi.org/10.1097/ IP.0000000000001343 (2016)| Moritz, K. J.et al. Eight hour continuous normothermic ex vivo kidney perfusion is a safe preservation technique for kidney transplantation: a new opportunity for the storage, assessment and repair of kidney grafts. Transplantation http://dx.doi.org/10.1097/ $\underline{\text { TP.0000000000001299 (2016) }}$ 